\title{
Acute biphenotypic leukaemia: immunophenotypic and cytogenetic analysis
}

\author{
Curtis A. Hanson, Mohamed Abaza, Susan Sheldon, Charles W. Ross, Bertram Schnitzer and \\ Luoyd M. STOolman Department of Pathology, University of Michigan Hospitals, Ann Arbor, Michigan
}

Received 28 July 1992; accepted for publication 3 November 1992

\begin{abstract}
Summary. The incidence of acute biphenotypic leukaemia has ranged from less than $1 \%$ to almost $50 \%$ in various reports in the literature. This wide variability may be attributed to a number of reasons including lack of consistent diagnostic criteria, use of various panels of antibodies, and the failure to recognize the lack of lineage specificity of some of the antibodies used. The morphology, cytochemistry, immunophenotype and cytogenetics of acute biphenotypic leukaemias from our institution were studied. The diagnostic criteria took into consideration the morphology of the analysed cells, light scatter characteristics, and evaluation of antibody fluorescence histograms in determining whether the aberrant marker expression was arising from leukaemic blasts or differentiated bone marrow elements. Fifty-two of 746 cases $(7 \%)$ fulfilled our criteria for acute biphenotypic leukaemias. These included 30 cases of acute lymphoblastic leukaemia (ALL) expressing myeloid antigens, 21 cases of
\end{abstract}

acute myelogenous leukaemia (AML) expressing lymphoid markers, and one case of ALL expressing both B- and T-cell associated antigens. The acute biphenotypic leukaemia cases consisted of four major immunophenotypic subgroups: CD2 + AML (11), CD19 + AML (8), CD13 and/or CD33+ ALL (24), CD11b + ALL (5) and others (4). Chromosomal analysis was carried out in $42 / 52$ of the acute biphenotypic leukaemia cases; a clonal abnormality was found in 31 of these 42 cases. This study highlights the problems encountered in the diagnosis of acute biphenotypic leukaemia, some of which may be reponsible for the wide variation in the reported incidence of this leukaemia. We suggest that the use of strict, uniform diagnostic criteria may help in establishing a more consistent approach towards diagnosis of this leukaemic entity. We also suggest that biphenotypic leukaemia is comprised of biologically different groups of leukaemia based on immunophenotypic and cytogenetic findings.
The classification of acute leukaemia has traditionally been based on a combination of morphology and cytochemical staining. These diagnostic modalities are quite sufficient to accurately classify the majority of acute leukaemias. Flow cytometric immunophenotyping has also evolved as an important laboratory tool to be used in the diagnosis and classification of acute leukaemia. By using monoclonal antibodies reactive against various lymphoid- or myeloidassociated antigens, it is possible to distinguish acute lymphoblastic leukaemia from acute myelogeneous leukaemia and to identify subsets within each of the respective acute leukaemias. In addition, immunophenotyping studies have given us insight into the immunologic sequence of normal lymphoid and myeloid ontogeny (LeBien \& Villablanca. 1990; Terstappen \& Loken, 1990).

Considerable interest has developed over the last decade in

Correspondence: Dr Curtis A. Hanson. University of Michigan Medical School, Department of Pathology/M5242 Med Sci I. 1301 Catherine Road, Ann Arbor, MI 48109-0602, U.S.A. those acute leukaemias that have immunophenotypes that do not fit into the accepted sequence of normal lymphoid or myeloid cell development (Perentesis et al, 1983; Mirro et al, 1985, 1986; Stass \& Mirro, 1985; Drexler et al, 1986; Campos et al, 1987; Cross et al, 1988; Schmitt-Graff et al, 1988; Del Vecchio et al, 1989; Kaplan et al, 1989; Akashi et al, 1990; Gerhartz \& Schmetzer, 1990; Sulak et al, 1990). Perhaps the most controversial and confusing of these immunologically aberrant cases have been the so-called acute biphenotypic leukaemias. A plethora of terminologies (hybrid, mixed-lineage, bilineal, biclonal, biphenotypic) has added to the confusion in this area (Gale \& Ben-Bassat, 1987; Maitreyan \& Gale, 1989). Such phenotypes imply that either malignant transformation leads to aberrant gene expression ('lineage infidelity') (Smith et al, 1983) or that co-expression of 'lineage-specific' markers occurs normally in differentiation of haematopoietic cells ('lineage promiscuity') (Greaves \& Chan, 1986; Greaves et al, 1986). The clinical significance of biphenotypia in acute leukaemia has not been determined, as such studies have suffered from the lack of consistent 
guidelines for classification. Some studies have suggested that biphenotypic leukaemias have a worse clinical prognosis than non-biphenotypic acute lymphoblastic leukaemia or acute myelogenous leukaemia, while other reports have not confirmed that clinical significance exists (Sobol et al, 1987; Ferrara et al, 1990; Pui et al, 1990; Bal et al, 1991; Wiersma et al, 1991).

This report describes 52 patients with acute biphenotypic leukaemia out of a cohort of 746 patients. The incidence of biphenotypia ( $7 \cdot 0 \%)$ in this study is lower than some studies in the literature have suggested, bringing into question the various criteria that have been used for the diagnosis of biphenotypic leukaemias. The 52 biphenotypic cases in this study are subcategorized into various immunophenotypic subgroups, which may prove helpful in understanding the biological sequence of haematopoietic development. Cytogenetic studies reveal a variety of chromosomal aberrations, in addition to the commonly reported $\mathrm{t}(9 ; 22)$ and $11 \mathrm{q} 23$ abnormalities (Hirsch-Ginsberg et al, 1988; Childs et al, 1988; Hayashi et al, 1990a, b). These cytogenetic studies are essential in characterizing these intriguing leukaemias and may provide additional diagnostic and biological information.

\section{MATERIALS AND METHODS}

Patient population. The case population for this study was derived from 746 specimens of adult and paediatric acute leukaemia analysed at the University of Michigan Flow Cytometry Laboratory from January 1985 to December 1990. A diagnosis of acute leukaemia was established using the morphologic and cytochemical criteria of the FrenchAmerican-British (FAB) cooperative group. Cytologic features were assessed on Wright-Giemsa-stained smears of bone marrow and/or peripheral blood. Cytochemical stains, including myeloperoxidase, Sudan black B, and alphanaphthyl butyrate esterase (non-specific esterase), were used to sub-classify the acute myelogenous leukaemia cases. Clinical information including age, sex, and mode of presentation (i.e., de novo versus relapse), was determined from data available to the laboratory.

Monoclonal antibodies. Surface immunophenotyping of leukaemic cells was performed by flow cytometry with the use of a broad panel of lymphoid- and myeloid-associated monoclonal antibodies (MoAbs). These included: B-cell associated MoAbs (CD19, CD20, CD22); T-cell-associated MoAbs (CD1, CD2, CD3, CD4, CD5, CD7, CD8); myeloid-associated MoAbs (CD11b, CD13, CD14, CD33); and non-lineage specific MoAbs (CD10, CD34 and HLA-DR).

Flow cytometric procedure. Mononuclear cells were isolated from heparinized blood and/or bone marrow specimens by standard Ficoll-Hypaque density gradient centrifugation. A Wright-Giemsa-stained cytocentrifuge preparation of the Ficoll-Hypaque-separated mononuclear cells was morphologically evaluated in each case. The percentage of blasts was determined on this preparation to evaluate the effectiveness of the gradient separation and to aid in the subsequent setting of light scatter gates. The mononuclear cells were analysed by either an Epics V or Profile Flow Cytometer (Coulter Corpora- tion, Hialeah, Fla.); 5000-10000 cells were analysed for each MoAb that was used. The mononuclear cells were then divided into 'electronic gates' based on the forward angle and $90^{\circ}$ light scatter characteristics of the various cell populations (Ross et al, 1990). Single-parameter, fluorescence histograms were then generated with each antibody for each of the gated cell populations. The percentage of cells positive for each marker was calculated by a normalized subtraction computer analysis program (IMMUNO; Coulter Corporation; Hialeah, Fla.) to account for the background and non-specific staining seen with an immunoglobulin isotype-matched negative control (Bagwell, 1989). Two-colour, direct immunofluorescent staining was performed in a limited number of cases using either a Profile or FACScan flow cytometer (Becton-Dickinson Corporation, Mountainview, Calif.). Interpretation of dual staining was based on simultaneous presence of red and green fluorescence.

Criteria for biphenotypic diagnosis. Biphenotypia was defined in this study as expression on leukaemic blasts of at least one lymphoid- and at least one myeloid-associated epitope. The diagnosis and identification of the acute biphenotypic leukaemias was a multi-step process based on various criteria. First, retrospective screening of a computer database was done to identify leukaemic cases that had greater than $15 \%$ positivity with at least one myeloid-associated (CD1 1b, CD13, CD14 or CD33) and one lymphoid-associated (CD1, CD2, CD3, CD5, CD8, CD19 or CD20) MoAb. CD4, CD7, CD10 and CD34 were not used as lineage-specific markers, as those antigens are commonly found in both lymphoid and myeloid cells (Hanson \& Gajl-Peczalska, 1988). The percentage of positivity was not the sole criterion used to define biphenotypia. This liberal screening threshold was selected to ensure that no potential cases were excluded. 131 cases were identified from the database by this initial screen.

All cases selected by this initial screen were then further evaluated for evidence of biphenotypia, using various morphologic and flow cytometric-derived criteria. These more strict criteria were used to address two issues: (1) to allow the consistent separation of leukaemic blasts from mature lymphocytes or differentiating myeloid (leukaemic or normal) cells based on a combination of flow cytometric light scatter characteristics, morphologic correlates, and percentages of antibody positivity; and (2) to allow the accurate determination of 'true' antigenic staining separate from non-specific background reactivity. This process included evaluation of the differential cell count to identify the types of cells being analysed by the flow cytometer. Light scatter (forward angle versus $90^{\circ}$ angle) characteristics were then used to establish electronic gates around the cell populations of interest. The resulting light scatter gates were correlated with the cytocentrifuge preparation differential in an attempt to determine which morphologic subsets were present in the respective gated areas. The light scatter distributions from these cases were also categorized according to the number of gated populations and whether the morphologic correlation suggested the presence of either a uniform population of blasts or mixtures of blasts and non-malignant cells in each of the gated areas.

To distinguish mature lymphoid cells from blasts, the 
finding of equal numbers of cells expressing multiple, pan-Tcell antigens (CD2, CD3, CD5, CD7 and CD4 and/or CD8) and the presence of comparable numbers of small lymphocytes on the differential count of gradient-separated cells were used as evidence that 'contaminating' lymphoid cells were in the leukaemic blast gate. A similar process was used to evaluate the possibility of differentiated myeloid cells contaminating the leukaemic blast gate.

The computer analysis program was used to subtract nonspecific background staining from true antigenic staining; this was, as expected, more of a problem in AML than in ALL cases. Computer programs for histogram analysis, whether based on simple integration or a subtraction algorithm, involve arbitrary settings of analytic parameters. Because of these arbitrary rules, falsely low or high percentages can be generated (Bagwell, 1989). Thus, it is essential to subjectively evaluate the flow cytometric histograms in comparison with the background reactivity to determine whether the calculated percentage of positivity accurately reflects the staining contribution of the negative control. If this evaluation suggested that the calculated percentages were in error, then a subjective determination was made as to whether or not the particular antigen in question was indeed being identified on the leukaemic cells.

Statistics. Statistical comparisons between the biphenotypic leukaemias and nonbiphenotypic leukaemias were performed using either the Student $t$ or Chi square analyses.

Cytogenetics. Heparinized bone marrow aspirates were cultured at $37^{\circ} \mathrm{C}$ at an approximate concentration of $10^{6}$ cells $/ \mathrm{ml}$ for 2,24 or $48 \mathrm{~h}$ in RPMI 1640 medium supplemented with $10 \%$ heat-inactivated fetal calf serum, penicillin and streptomycin. When sufficient specimen was available, duplicate cultures were established for 24-48 h synchronization using fluorodeoxyuridine. Cells were arrested in meta- phase with colcemid, harvested, and fixed; slides were prepared using standard cytogenic techniques (Sheldon \& Nichols, 1981). Trypsin-Giemsa banding was used. A minimum of 20 cells were analysed in the majority of cases. Cytogenetic findings were described using the International Standards for Cytogenic Nomenclature (ISCN) nomenclature (Harnden \& Klinger, 1985).

\section{RESULTS}

\section{Incidence/excluded cases}

The Flow Cytometry Laboratory analysed 746 cases of acute leukaemia between the years 1985 and 1990. 131 cases were identified on our initial screen for biphenotypia based solely on percentage $(>15 \%)$ of antibody positivity. Seventynine of these 131 cases were not included in our final biphenotypic group; 50 of these were acute myelogenous leukaemias (AML) and 29 were acute lymphocytic leukaemias (ALL). The percentage of blasts on the cytocentrifuge preparation of those cases ranged from $25 \%$ to $100 \%$ with a median of $72 \%$ blasts. The remaining 52 cases $(7 \cdot 0 \%)$ fulfilled our criteria for biphenotypic leukaemia; 21 were AML and 31 were ALL. The blast percentage on the cytocentrifuge preparation in the biphenotypic cases ranged from $26 \%$ to $100 \%$ with a median of $76 \% ; 10$ cases had less than $50 \%$ blasts.

Four basic light scatter patterns emerged when analysing the leukaemic specimens of both the biphenotypic cases and the cases excluded from the study: (1) a single uniform population composed entirely of leukaemic blasts; (2) a single population composed of a mixture of blasts and lymphocytes and/or differentiated myeloid cells; (3) two or more distinct populations, in which one uniform blast population could be clearly separated from normal lymphocytes or differentiated

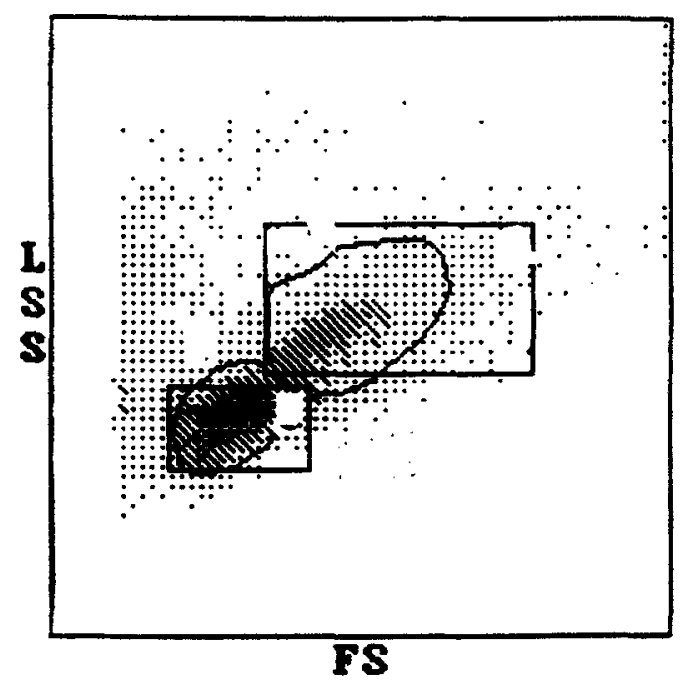

(A)

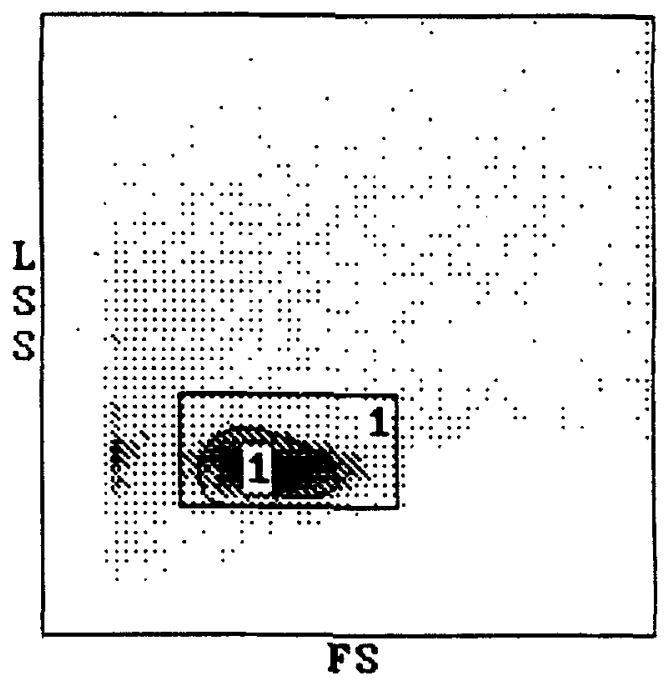

(B)

Fig 1. (A) A dot plot of forward scatter (FS) and orthogonal scatter (LSS) of an acute biphenotypic leukaemia with a single light scatter population [patterns (1) and (2) from text]. The rectangle around the cell cluster is machine-generated. (B) A dot plot of forward scatter (FS) and orthogonal scatter (LSS) of an acute biphenotypic leukaemia with two light scatter populations [patterns (3) and (4) from text]. A higher percentage of cells is seen in the population with low forward and orthogonal scatter. The rectangle is machine-generated, while the ellipse is operator-generated. 
Table I. Clinical features.

\begin{tabular}{lccc}
\hline & AML $(n=21)$ & ALL $(n=31)$ & Total $(n=52)$ \\
\hline Frequency (\%) & $7 \cdot 0$ & $6 \cdot 9$ & $7 \cdot 0$ \\
Age (years) & & & \\
$\quad<2$ & 0 & 0 & 0 \\
$2-8$ & 2 & 17 & 19 \\
$9-18$ & 3 & 7 & 10 \\
$>19$ & 16 & 7 & 23 \\
Sex & & & \\
Male & 12 & 15 & 27 \\
Female & 9 & 16 & 25 \\
Presentation & & & \\
De novo & 18 & 19 & 37 \\
Relapse & 0 & 11 & 11 \\
Blast crisis & 3 & 1 & 4 \\
\hline
\end{tabular}

Table II. Lymphoid antigen-positive AML $(n=21)$.

\begin{tabular}{llll}
\hline & CD2 $+(n=11)$ & CD19+(n=8) & CD20 $+(n=2)$ \\
\hline Age (years) & & 2 & \\
$2-8$ & 0 & 1 & 0 \\
$9-18$ & 2 & 5 & 2 \\
$>18$ & 9 & & \\
FAB & & 0 & 0 \\
M1 & 0 & 6 & 0 \\
M2 & 0 & 1 & 0 \\
M3 & 2 & 0 & 0 \\
M4 & 4 & 1 & 2 \\
M5 & 2 & 0 & 0 \\
M6 & 0 & 0 & 0 \\
M7 & 0 & & \\
Presentation & & 8 & 2 \\
De novo & 8 & 0 & 0 \\
Relapse & 0 & 0 & \\
Blast crisis & 3 & & $28-64$ \\
Positivity & & $42-80$ & 46 \\
Range (\%) & $34-78$ & 52 & \\
Median (\%) & 56 & & \\
\hline
\end{tabular}

myeloid cells; and (4) two or more light scatter populations, with at least one area composed of a mixture of blasts and lymphocytes and/or myeloid cells (Fig 1). The majority (37/ 52) of the biphenotypic cases had uniform light scatter populations of blasts easily separated from non-blast populations. The excluded group showed more variable light scatter findings. Forty of the 79 excluded cases were deleted due to the finding of mature T-lymphocytes intermixed with the leukaemic blasts in the same light scatter population; 11 cases were excluded based on myeloid cell contamination with lymphoid blasts. These cases demonstrating a mixture of blasts and mature lymphoid or myeloid elements could have been called biphenotypic if only percentages of positivity were considered; for example, T-lymphocytes plus myeloblasts could be mistakenly called T-lymphoid antigen-positive AML. The remaining cases were excluded based on the failure of the histogram analysis program to calculate percentages of positivity that reflected the separation of true staining from non-specific background staining based on subjective histogram evaluations.

The annual frequency of biphenotypia was consistent over the first 5 years of the study $(0 \cdot 5-8 \cdot 0 \%)$, but increased to $13 \%$ of all acute leukaemias during $1990(P>0 \cdot 05) .21 / 299$ $(7 \cdot 0 \%)$ acute myelogenous leukaemia (AML) cases, diagnosed by FAB criteria, expressed a lymphoid-associated marker. These 21 cases of AML included six with the morphologic diagnosis of FAB-M2, three with FAB-M3, four with FAB-M4, five with FAB-M5, and three cases were CML in myeloid blast crisis. $30 / 447$ (6.9\%) acute lymphoblastic leukaemia (ALL) cases, diagnosed morphologically, expressed myeloid-associated marker(s) by flow cytometry. One case of chronic myelogenous leukaemia (CML) in lymphoid blast crisis was included in the biphenotypic ALL subgroup. One additional ALL case was also identified that expressed both B- and T-cell-associated antigens, but no myeloid antigens.

\section{Demographic}

Table I summarizes the age distribution and clinical presentation of the biphenotypic AML and ALL groups. The majority of biphenotypic leukaemias in childhood had ALL morphology while the majority of adult biphenotypic leukaemias were diagnosed as AML. All 11 cases of acute biphenotypic leukaemia in relapse were ALL by FAB criteria. Ten of these 11 were initially B-cell precursor ALL, while one case was initially diagnosed as a T-ALL. No aberrant myeloid reactivity was immunologically evident in any of these 11 cases at the time of initial presentation.

Lymphoid antigen-positive AML

The diagnosis of AML was confirmed by the predominant immunophenotypic expression of myeloid-associated antigens. All 21 cases of biphenotypic leukaemia morphologically classified as AML (lymphoid antigen-positive AML) expressed $\operatorname{CD} 13(n=18), \operatorname{CD} 33(n=16)$ (Fig 2A), and/or CD14 $(n=7)$ and were further subgrouped into CD2-positive AML ( $n=11$ cases), CD19-positive AML ( $n=8$ cases), and CD20-positive AML ( $n=2$ cases) (Table II). It is of interest to note that the FAB diagnoses in the CD2-positive AML group included six cases with a monocytic component (FAB-M4 or FAB-M5), two acute promyelocytic leukaemias (FAB-M3), and three $C M L$ cases in myeloid blast crisis. In contrast, six of the eight CD19-positive AML group were FAB-M2. The per cent positivity of $\mathrm{CD} 2, \mathrm{CD} 19$ and $\mathrm{CD} 20$ in these lymphoid antigen-positive AML cases clearly indicated that a significant number of the leukaemic cells expressed these antigens. However, it can also be seen from these percentages (Table II) that not every single leukaemic cell within a case coexpressed detectable myeloid- and lymphoid-associated antigens. This implies that either various immunologic subclones 
Table III. Myeloid antigen-positive ALL $(n=31)$.

\begin{tabular}{|c|c|c|c|c|c|c|}
\hline & $\begin{array}{l}\text { CD33+ only } \\
(n=11)\end{array}$ & $\begin{array}{l}\mathrm{CD} 13+/ \mathrm{CD} 33+ \\
(n=9)\end{array}$ & $\begin{array}{l}\text { CD11b+ } \\
(n=5)\end{array}$ & $\begin{array}{l}\text { CD13+ only } \\
(n=4)\end{array}$ & $\begin{array}{l}\text { CD14+ } \\
(n=1)\end{array}$ & $\begin{array}{l}\mathrm{CD} 2+ \\
(n=1)\end{array}$ \\
\hline \multicolumn{7}{|l|}{ Age (years) } \\
\hline $2-8$ & 4 & 5 & 3 & 3 & 1 & 1 \\
\hline $9-18$ & 4 & 2 & 0 & 1 & 0 & 0 \\
\hline$>18$ & 3 & 2 & 2 & 0 & 0 & 0 \\
\hline \multicolumn{7}{|c|}{ Immunophenotype } \\
\hline B-precursor & 9 & 9 & 3 & 3 & 1 & 1 \\
\hline T-cell & 2 & 0 & 2 & 1 & 0 & 0 \\
\hline \multicolumn{7}{|l|}{ Presentation } \\
\hline De novo & 6 & 5 & 4 & 2 & 1 & 1 \\
\hline Relapse & 5 & 3 & 1 & 2 & 0 & 0 \\
\hline Blast crisis & 0 & 1 & 0 & 0 & 0 & 0 \\
\hline \multicolumn{7}{|l|}{ Positivity } \\
\hline Range $(\%)$ & $19-68$ & $21-87 / 17-58$ & $23-56$ & $29-44$ & 54 & 71 \\
\hline Median (\%) & 32 & $53 / 34$ & 39 & 39 & - & - \\
\hline
\end{tabular}

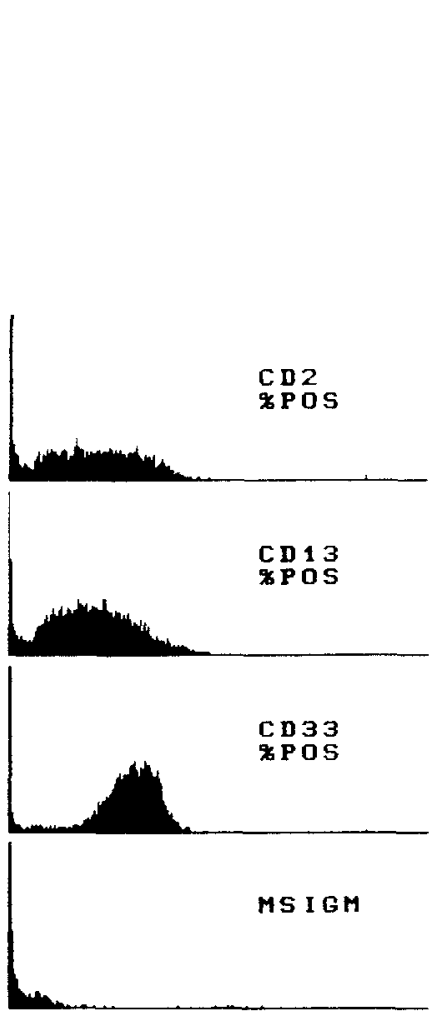

(A)

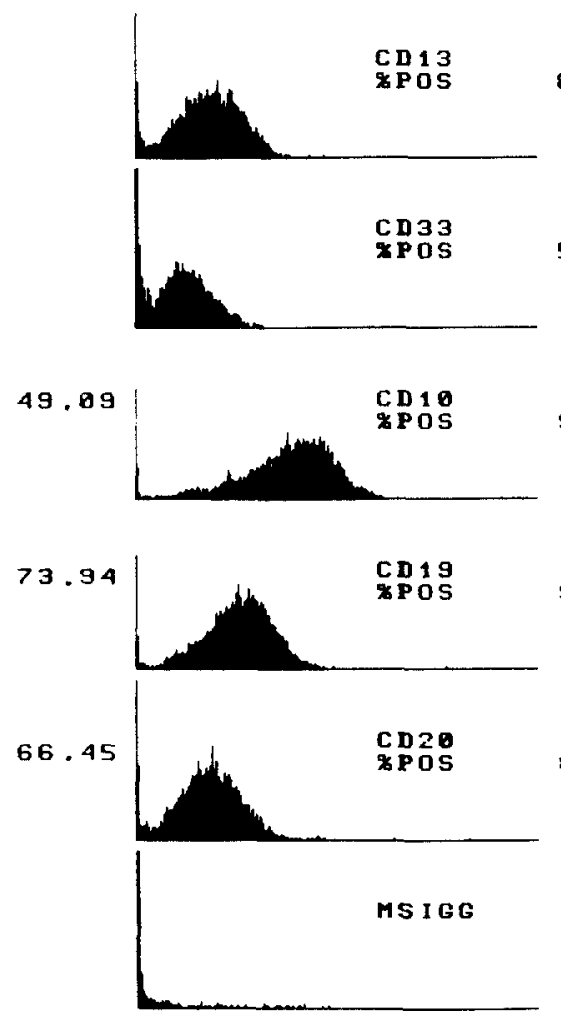

(B)
87.22

58.09

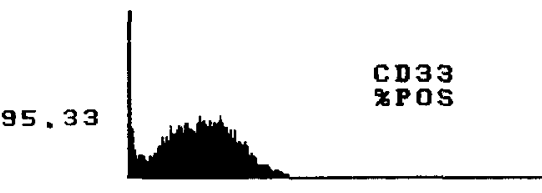

68.09

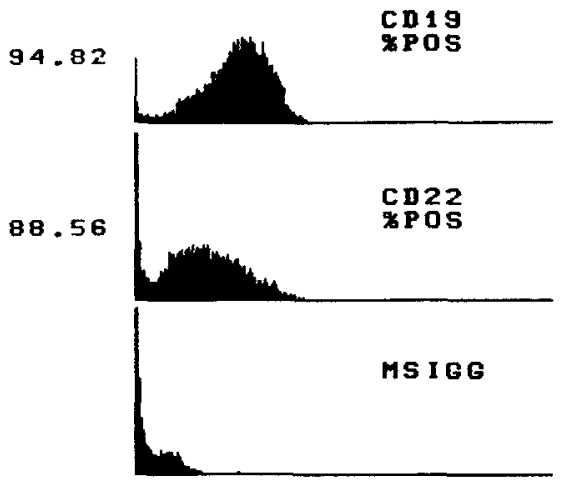

(C)

Fig 2. (A) Single-parameter fluorescence histograms of $\mathrm{CD} 2, \mathrm{CD} 13, \mathrm{CD} 33$, and mouse isotype-matched negative control antibody in a case of CD2 + AML. (B) Single-parameter fluorescence histograms of CD13, CD33, CD10, CD19, CD20, and mouse isotype-matched negative control antibody in a $\mathrm{CD} 13 / \mathrm{CD} 33+\mathrm{ALL}$. (C) Single-parameter fluorescence histograms of CD33, CD19, CD22, and mouse isotype-matched negative control in a CD 33 + ALL. The percentage of positive-staining cells is shown to the right of the histogram.

were present, some biphenotypic and some not, or that the lymphoid-associated antigen was too weakly expressed in some of the leukaemic cells to be detected beyond background staining.
Myeloid antigen-positive ALL

The 31 cases of biphenotypic ALL (Table III) included 26 cases of B-precursor ALL, expressing CD10, CD19, CD20 and/or $\mathrm{CD} 22$, and five cases which had T-cell antigen 
Table IV. Comparison of fluorescence intensity of 'aberrant' marker in biphenotypic and non-biphenotypic leukaemia.

\begin{tabular}{|c|c|c|c|c|c|c|c|c|}
\hline \multicolumn{4}{|l|}{ Biphenotypic } & \multicolumn{4}{|c|}{ Non-biphenotypic } & \multirow[b]{3}{*}{$P \ddagger$} \\
\hline \multirow[b]{2}{*}{ Classification } & \multirow[b]{2}{*}{$n$} & \multicolumn{2}{|l|}{ MCFI* $^{*}$} & \multirow[b]{2}{*}{ Classification } & \multirow[b]{2}{*}{$n$} & \multicolumn{2}{|l|}{ MCFI* } & \\
\hline & & Median & Range & & & Median & Range & \\
\hline CD19+ ${ }^{+}$AML & 8 & 59 & $52-65$ & B-pre ALLt & 253 & 75 & $30-130$ & $<0.01$ \\
\hline $\mathrm{CD} 2+\mathrm{AML}$ & 11 & 73 & $65-80$ & T-ALL & 26 & 97 & $72-126$ & $<0.00$ \\
\hline $\mathrm{CD} 13^{+} \mathrm{ALL}$ & 13 & 56 & $31-67$ & AML & 196 & 76 & $51-118$ & $<0 \cdot 01$ \\
\hline $\mathrm{CD} 33^{+} \mathrm{ALL}$ & 20 & 47 & $32-61$ & AML & 195 & 76 & $47-116$ & $<0.01$ \\
\hline
\end{tabular}

${ }^{*} \mathrm{MCFI}=$ mean channel fluorescence intensity.

$\uparrow$ B-pre ALL=B-precursor ALL.

$\ddagger$ Student $t$ test analysis.

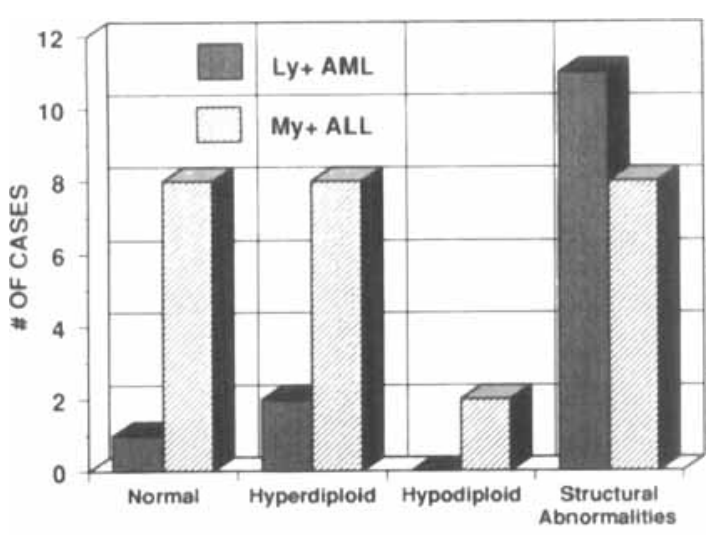

CYTOGENETIC FINDINGS

Fig 3. Bar graph showing distribution of general cytogenetic abnormalities in lymphoid antigen-positive AML (Ly+AML) and myeloid antigen-positive ALL (My + ALL) cases.

expression. The B-precursor ALL cases were further subclassified into CD13-positive/CD33-positive ALL ( $n=9$ cases) (Fig $2 \mathrm{~B}$ ), CD33 only-positive ALL ( $n=9$ cases) (Fig $2 \mathrm{C}$ ), CD11bpositive ALL ( $n=3$ cases), CD13 only-positive ALL ( $n=3$ cases) and C14-positive ALL $(n=1$ case). One B-precursor ALL case co-expressed the T-cell-associated antigen, CD2.

\section{Fluorescence intensity}

Table IV compares the median fluorescence intensity (FI) of the aberrant lymphoid or myeloid marker in the biphenotypic leukaemias to the median FI of that same marker expressed in non-biphenotypic acute leukaemia. The FI of CD2. CD19, CD1 3 and CD 33 in the biphenotypic leukaemias was significantly less (2-3 times dimmer) than the FI of these antigens in the corresponding non-biphenotypic leukaemias (T-ALL, B-precursor ALL, and AML, respectively).

\section{CD34 expression}

The CD34 antigen, which can be identified in haematopoietic progenitor cells, is a characteristic marker of cells in the earliest stages of lymphoid and haematopoietic development (Civin et al, 1987). Expression of the CD34 epitope on leukaemic cells suggests that malignant transformation occurred at an early stage of haematopoietic development. Conversely, the lack of expression may reflect transformation later in ontogeny. We thus evaluated CD34 expression in the biphenotypic acute leukaemias to determine whether these cases were indeed more 'primitive' (i.e. having CD34 expression) than non-biphenotypic acute leukaemias diagnosed in our laboratory.

CD34 expression was significantly higher in biphenotypic leukaemias than in non-biphenotypic leukaemias. $31 / 42$ (74\%) cases evaluated were CD34-positive. In contrast. $48 \%$ of all non-biphenotypic acute leukaemias from our laboratory expressed CD34 $(P<0 \cdot 05) .16 / 19$ (84\%) lymphoid antigen-positive AML and 15/23 (65\%) myeloid-antigenpositive ALL cases were positive for CD34. This compares with $49 \%$ of AML $(P<0.05)$ and $46 \%$ of ALL $(P<0.05)$ cases expressing CD34 in the non-biphenotypic acute leukaemia group analysed in our laboratory. No difference in CD34 antigenicity was seen among the various age groups or between de novo and relapse presentations.

\section{Cytogenetics}

Cytogenetic analysis was carried out in $42 / 52(81 \%)$ of the biphenotypic acute leukaemia cases; these included 16 cases of AML and 26 cases of ALL. A normal, 46,XX female or $46, \mathrm{XY}$ male karyotype was found in $11 / 42(26 \%)$ of the biphenotypic cases, including 2/16 (12\%) AMLs and 9/26 (35\%) ALLs. A clonal abnormality was found in $31 / 42$ (74\%) cases, including hypodiploidy, hyperdiploidy, and structural abnormalities (Fig 3). These chromosomal abnormalities were found in both ALLs and AMLs in a distribution roughly similar to that found in acute non-biphenotypic leukaemias.

Fig 4 and Table $V$ demonstrate that a relatively limited number of cytogenetic aberrations were evident in the lymphoid antigen-positive AML group. Several of the chromosomal abnormalities found in this group were classical AML-associated translocations (Arthur et al, 1989; LeBeau, 1990). These included: six $t(8 ; 21)(\mathrm{q} 22: \mathrm{q} 22)$; three $\mathrm{t}(9 ; 22)(\mathrm{q} 34 ; \mathrm{q} 11)$ (Philadelphia chromosome); two 


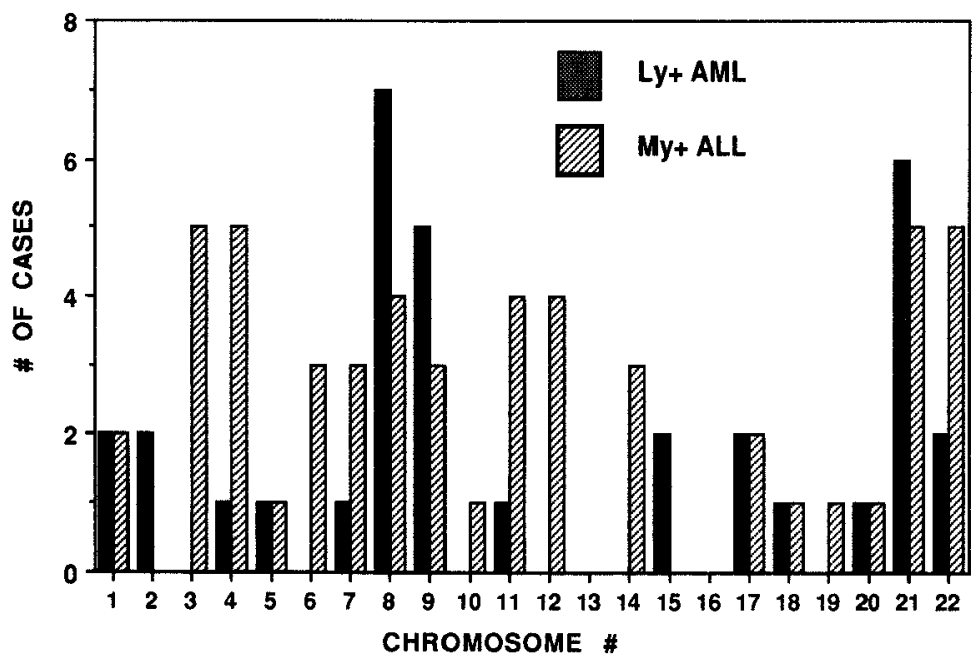

Fig 4. Bar graph showing distribution of specific cytogenetic abnormalities for each chromosome in lymphoid antigen-positive AML ( $\mathrm{Ly}+\mathrm{AML})$ and myeloid antigen-positive ALL (My + ALL).

Table V. Cytogenetic findings in lymphoid antigen-positive AML.

\begin{tabular}{|c|c|c|c|}
\hline & Karyotype & FAB & $\mathrm{D} / \mathrm{R} / \mathrm{BC}^{*}$ \\
\hline \multirow[t]{9}{*}{$\mathrm{CD} 2+\mathrm{AML}(n=9)$} & $50, X Y,+2,+4,+8,+20$ & M4 & $\mathrm{D}$ \\
\hline & $46, X X, \operatorname{inv}(16)(p 13 q 22)$ & $\mathrm{M} 4 \mathrm{e}$ & $\mathrm{D}$ \\
\hline & $46, \mathrm{XX}, \mathrm{t}(9 ; 22)(\mathrm{q} 34 ; \mathrm{q} 11)$ & $\ldots$ & $\mathrm{BC}$ \\
\hline & $46, \mathrm{XY}, \mathrm{t}(9 ; 22)(\mathrm{q} 34 ; \mathrm{q} 11)$ & - & $\mathrm{BC}$ \\
\hline & $46, \mathrm{XY}, \mathrm{t}(9 ; 22)(\mathrm{q} 34: \mathrm{q} 11)$ & - & $\mathrm{BC}$ \\
\hline & $46, X Y, t(15 ; 17)(q 32 ; q 17)$ & $\mathrm{m} 3$ & $\mathrm{D}$ \\
\hline & $46, X X,(t ? ; 22)$ & M3 & $\mathrm{D}$ \\
\hline & $46, \mathrm{XX}$ & M5 & $\mathrm{D}$ \\
\hline & $46, \mathrm{XX}$,(pending) & M4 & $\mathrm{D}$ \\
\hline \multirow[t]{7}{*}{$\operatorname{CD} 19+\operatorname{AML}(n=7)$} & $46, \mathrm{XY}, \mathrm{t}(8 ; 21)(\mathrm{q} 22 ; \mathrm{q} 22)$ & M2 & $\mathrm{D}$ \\
\hline & $47, \mathrm{XX}, \mathrm{t}(8 ; 21)(\mathrm{q} 22 ; \mathrm{q} 22),+18, \operatorname{del}(9)(\mathrm{q} 13 ; 22)$ & M2 & $\mathrm{D}$ \\
\hline & $46, X X, t(8 ; 21)(q 22 ; q 22)$ & $\mathrm{M} 2$ & $\mathrm{D}$ \\
\hline & $46 . X Y, t(8 ; 21)(\mathrm{q} 22 ; \mathrm{q} 22)$ & M2 & $\mathrm{D}$ \\
\hline & $46, X Y, t(8: 21)(q 22 ; q 22), \operatorname{del}(9)(\mathrm{q} 22)$ & M2 & $\mathrm{D}$ \\
\hline & $46, \mathrm{XX}, \mathrm{t}(8 ; 21)(\mathrm{q} 22 ; \mathrm{q} 22), \mathrm{t}(1 ; 15)(\mathrm{p} 15 ; \mathrm{q} 22), \mathrm{t}(7 ; 9)(\mathrm{p} 13 ; \mathrm{q} 22)$ & M2 & $\mathrm{D}$ \\
\hline & $46 . \mathrm{XX}$ & M5 & $\mathrm{D}$ \\
\hline $\operatorname{CD} 20+\operatorname{AML}(n=1)$ & $46, X Y, t(2 ; 11), \operatorname{del}(5)(q)$ & M5 & $\mathrm{D}$ \\
\hline
\end{tabular}

* $\mathrm{D}=$ de novo $\mathrm{R}=$ relapse; $\mathrm{BC}=$ blast crisis.

acute promyelocyte leukaemias (FAB-M3) with a $\mathrm{t}(15 ; 17)(\mathrm{q} 22 ; \mathrm{q} 21)$; one translocation involving 11q23; one acute myelomonocytic leukaemia with eosinophilia (FABM4e) having an inv(16)(p13q22); one trisomy 8; and one case of $5 \mathrm{q}-$. Interestingly, all six $\mathrm{t}(8 ; 21)$ cases expressed CD19 as the aberrant lymphoid-associated antigen. No typical ALL-associated translocations were identified.

The myeloid antigen-positive ALLs, in contrast to the AMI group, showed a wide range of chromosomal abnormalities affecting multiple chromosomes (Fig 4 and Table VI). Trisomy 21 was identified in four cases; in only one of these cases did the patient have Down's syndrome. Other common abnormalities included: three $\mathrm{t}(9 ; 22)$ (Philadelphia chromosome): three with trisomy 8; three with translocations involving 3q21; three cases involving $17 \mathrm{q}$; and two $11 \mathrm{q} 23$ translocations, as well as several others. It is interesting to note that some of these abnormalities such as trisomy 21 , trisomy 8 , and translocations involving chromosomes $3 \mathrm{q} 21$ or $17 \mathrm{q}$, are more commonly found in myeloid processes than in ALL (Arthur et al, 1989; LeBeau et al, 1990). However, there were too few cases in this study to permit determination of statistical significance.

\section{DISCUSSION}

In this study we have retrospectively analysed the immunophenotype of 746 cases of acute leukaemia studied in our laboratory. From this cohort, we have identified 52 acute biphenotypic leukaemias $(7 \cdot 0 \%)$, with lymphoid antigenpositive AML and myeloid antigen-positive ALL diagnosed in 
Table VI. Cytogenetic findings in myeloid antigen-positive ALL.

\begin{tabular}{|c|c|c|c|}
\hline & Karyotype & $\mathrm{B} / \mathrm{T}^{*}$ & $\mathrm{D} / \mathrm{R} / \mathrm{BC} t$ \\
\hline \multirow[t]{10}{*}{ CD33 + ALL $(n=10)$} & $47, X X,+17(q), \operatorname{del}(11)(q 21), 19 q+$ & $\mathrm{B}$ & $\mathrm{R}$ \\
\hline & $46, \mathrm{XX},-8,+$ mar & $\mathrm{B}$ & $\mathrm{R}$ \\
\hline & $45, \mathrm{XY},-20, \mathrm{t}(\mathrm{q}: 22)(\mathrm{q} 34 ; \mathrm{q} 11)$ & $\mathrm{B}$ & $\mathrm{R}$ \\
\hline & $46, \mathrm{XX}$ & $\mathrm{B}$ & $\mathrm{D}$ \\
\hline & $53, \mathrm{XX}$ & $\mathrm{B}$ & $\mathrm{D}$ \\
\hline & $46, \mathrm{XY} ; 86-89, \mathrm{XXYY},+1$ & $\mathrm{~B}$ & $\mathrm{D}$ \\
\hline & $46, \mathrm{XX}$ & $\mathrm{B}$ & $\mathrm{R}$ \\
\hline & $45, X,-7,+\mathrm{i}(17)(\mathrm{q}), \mathrm{t}(11 ; 14),(\mathrm{q} 23 ; \mathrm{q} 32),+\operatorname{der}(1) \mathrm{t}(1 ; ?)$ & $\mathrm{B}$ & $\mathrm{R}$ \\
\hline & $46, \mathrm{XY}$ & $\mathrm{T}$ & $\mathrm{D}$ \\
\hline & $47, \mathrm{XY},+19$ & $\mathrm{~T}$ & $\mathrm{D}$ \\
\hline \multirow[t]{7}{*}{$\mathrm{CD} 13+/ 33+\mathrm{ALL}(n=7)$} & $45, X, t(3 ; 12)(\mathrm{q} 21 ; \mathrm{p} 13), \mathrm{t}(5 ; 12)(\mathrm{q} 21 ; \mathrm{p} 13)$ & $\mathrm{B}$ & $\mathrm{R}$ \\
\hline & $47, \mathrm{XX},-4,-8,-18,-21,+17, \operatorname{der}(4), \operatorname{der}(8), \operatorname{der}(21)(\mathrm{p} 11), \mathrm{t}(3: 7)(\mathrm{p} 14 ; \mathrm{p} 22)+\operatorname{mar}$ & $\mathrm{B}$ & $\mathrm{R}$ \\
\hline & $46, X Y$ & $\mathrm{~B}$ & $\mathrm{D}$ \\
\hline & $47, X Y,+21, t(7 ; 12)(\mathrm{p} 12 ; \mathrm{p} 11), \operatorname{del}(4)(\mathrm{q} 11)$ & $\mathrm{B}$ & $\mathrm{R}$ \\
\hline & $46, X Y, t(9 ; 22)(\mathrm{q} 34 ; \mathrm{q} 11)$ & $\mathrm{B}$ & $\mathrm{D}$ \\
\hline & $48-52, X Y$ & $\mathrm{~B}$ & $\mathrm{D}$ \\
\hline & $46, X Y$ & $\mathrm{~B}$ & $\mathrm{D}$ \\
\hline \multirow[t]{3}{*}{ CD11b + ALL $(n=3)$} & $47, \mathrm{XX},+21,53-56, \mathrm{XX},+21$ & $\mathrm{~B}$ & $\mathrm{D}$ \\
\hline & $46, X Y$ & $\mathrm{~B}$ & $\mathrm{D}$ \\
\hline & $52, \mathrm{XX},+8,+6,+14,+17,+21,+21$ & $\mathrm{~B}$ & $\mathrm{D}$ \\
\hline \multirow[t]{4}{*}{$\operatorname{CD} 13+\operatorname{ALL}(n=4)$} & $46, \mathrm{XX}$ & $\mathrm{B}$ & $\mathrm{R}$ \\
\hline & $46, X Y$ & $\mathrm{~B}$ & $\mathrm{D}$ \\
\hline & $46, X X,-11,+\operatorname{der}(11), \mathrm{t}(3 ; 11)(\mathrm{q} 21 ; \mathrm{q} 23), \operatorname{dup}(3)(\mathrm{q} 21), \mathrm{t}(9 ; 22)(\mathrm{q} 34 ; \mathrm{q} 11)$ & $\mathrm{B}$ & $\$$ \\
\hline & $47, \mathrm{XX},-8,-18,-18,-22,+4,+\operatorname{mar}(3), \operatorname{del}(3)(\mathrm{q} 11), \mathrm{t}(3 ; ?)(\mathrm{p} 12 ; 3)$ & $\mathrm{T}$ & $\mathrm{D}$ \\
\hline $\mathrm{CD} 14+\operatorname{ALL}(n=1)$ & $59, \mathrm{XX},+4,+6,+8,+10,+11,+12,+14,+17,+18,+21,+21,+22$ & $\mathrm{~B}$ & $\mathrm{D}$ \\
\hline $\operatorname{CD} 2+\operatorname{ALL}(n=1)$ & $46, \mathrm{XY}$ & $\mathrm{B}$ & $\mathrm{D}$ \\
\hline
\end{tabular}

${ }^{*} \mathrm{~B}=\mathrm{B}$-precursor ALL; $\mathrm{T}=\mathrm{T}$-ALL.

$\mathrm{D}=$ de novo; $\mathrm{R}=$ relapse; $\mathrm{BC}=$ blast crisis.

roughly the same percentage of cases. The difficulty in evaluating these results is that the reported frequency of biphenotypic leukaemia in the literature has varied from less than $1 \%$ of acute leukaemia cases in some studies to almost $50 \%$ of acute leukaemia cases in others (Perentesis et al, 1983: Mirro et al, 1985, 1986; Stass \& Mirro, 1985; Drexler et al, 1986; Campos et al, 1987; Cross et al, 1988; SchmittGraff et al, 1988; Del Vecchio et al, 1989; Kaplan et al, 1989; Akashi et al, 1990; Gerhartz \& Schnitzer, 1990; Sulak et al, 1990). This widely variably incidence range cannot be rationally explained solely on the basis of clinical variability between different population groups. Indeed, this variability along with the lack of consensus as to clinical or biological significance of biphenotypia in acute leukaemia is undoubtedly due to several contributing factors: the use of inconsistent and variable diagnostic criteria, the inevitable (but frequently ignored) subjectiveness of immunophenotypic interpretation, and the lack of a standardized and uniform panel of monclonal antibodies utilized in immunophenotypic analyses (Gale \& Ben-Bassat, 1987; Maitreyan \& Gale, 1989).

The lack of an accepted, standardized, and reproducible method of immunophenotypic analysis and the subjectiveness of flow cytometric interpretation are probably the biggest contributors to the inconsistency of diagnosing biphenotypia. The single diagnostic criterion listed in several reports has been the simple requirement that only $20 \%$ or $30 \%$ of cells needs to express either lymphoid- or myeloidassociated antigens to meet the definition of biphenotypia (Perentesis et al, 1983; Mirro et al, 1985, 1986; Stass \& Mirro, 1985; Campos et al, 1987; Cross et al, 1988; Schmitt-Graff et al, 1988; Del Vecchio et al, 1989; Akashi et al, 1990; Gerhartz \& Schnitzer, 1990; Sulak et al, 1990). The use of this limited definition fails to recognize the problems associated with analysing blood or bone marrow specimens that inevitably contain a mixture of leukaemic blasts and mature lymphocytes or differentiating myeloid (leukaemic or normal) cells. In this same context, it must also be remembered that the patient specimen received for flow cytometric analysis may contain a different mixture of blasts and normal blood/bone marrow elements than the specimen received for morphologic review. We have tried to overcome this variability by examining the morphology of each specimen received in the laboratory, as well as preparing and examining a cytocentri- 
fuge preparation of the gradient-separated cells. This latter methodology allowed us to correlate directly the morphologic features of the specimen with the subsequent light scatter characteristics to permit the establishment of accurate electronic 'gates' around the leukaemic cells of interest. By ignoring these morphologic correlates, one may interpret the flow cytometric results erroneously if they are based solely on orthogonal and forward light scatter characteristics. Normal lymphocytes, erythroid precursors, or differentiating myeloid cells may fall into the same gated area as the leukaemic blasts and must be distinguished from them and excluded to accurately interpret the immunophenotypic results.

The morphologic features, the cytocentrifuge preparation of gradient-separated cells, the percentage of blasts present, the light scatter characteristics, and the fluorescence histogram patterns, etc., all potentially provide significant diagnostic information that far outweighs the diagnostic significance of arbitrary percentages of stained cells. Although it is likely that previous studies of biphenotypia have informally used such subjective criteria, the failure to fully document and discuss these diagnostic criteria has presented the development of a uniform and consistent method of analysing and diagnosing biphenotypic leukaemias. Our approach may not necessarily be the ideal method, but it does provide a starting point for more accurate comparison.

Two-colour immunofluorescence has rapidly become the method of choice for analysing clinical specimens (Shapiro, 1988: Uckun et al, 1989). This is an ideal method for demonstrating simultaneous expression of antigens, such as in biphenotypia, especially when leukaemic blasts are intermixed with normal or differentiating cells. Because our study was retrospectively based over a 6-year period, we were restricted to using single-colour immunophenotyping results. It is presumptuous to assume that this latter technique is outdated and undesirable and that two-colour analysis is the absolute gold standard. Although determination of dual staining by single-colour analysis does require rigorous criteria, the use of two-colour immunofluorescence staining does not preclude the necessity of utilizing morphologic, light scatter, and fluorescence histogram data when interpreting results. Indeed, the interpretation of flow cytometric 'positivity' with two-colour staining should be based on the same strict criteria that we have used in this study. Moreover, the use of directly conjugated MoAbs in twocolour analysis may decrease sensitivity, potentially up to 5 6-fold (Shapiro, 1988). The fluorescence intensity of the aberrantly expressed antigens was 2-3 times dimmer in the biphenotypic leukaemias, theoretically making them more difficult to detect by direct-staining methods. In addition, two-colour staining requires adjusting colour compensation and setting quadrant gates, adding further subjectivity to the flow cytometric process. In spite of these considerations, the evolution of two-colour immunofluorescence potentially will promote greater specificity of immunophenotyping and a better understanding of normal haematopoietic development.

Another important factor that has contributed to the confusion in the literature about biphenotypia has been the use of widely variable panels of monoclonal antibodies.
Fortunately, the development of the cluster designation (CD) system for categorizing monoclonal antibody reactivity provides a more consistent method of evaluating and comparing antibodies (Reinherz, 1986; McMichal, 1987; Knapp, 1989). In addition, it is also important to consider the possibility that many of the MoAbs used diagnostically may not be lineagespecific, but rather lineage-associated (Reinherz, 1986: Campos et al, 1987; McMichal, 1987; Hanson \& Gajl-Peczalska. 1988; Knapp, 1989). In other words, insufficient knowledge about the specificity of monoclonal antibody reactivity may prevent accurate lineage determination (Greaves et al, 1986).

In this study, biphenotypia was based on CD2, CD19 or CD20 expression in AML and CD11b, CD13, CD14 and/or CD33 expression in ALL. These antibodies were chosen based on two factors: one, they have historically been considerd as lineage-restricted; and two, they have been used as evidence of biphenotypia in previous studies. These arbitrary designations have been determined by time-honoured, single-colour studies of haematopoietic development in both normal bone marrows and in acute leukaemias and may not survive the test of time as more sophisticated flow cytometric studies emerge. Reports have now described CD2 expression in fetal non-T-cells (Uckun et al, 1989), CD19 and CD20 in myeloid cells (Campos et al, 1987: Gerhartz \& Schnitzer, 1990), and CD14 in significant numbers of malignant lymphomas (Medeiros et al, 1991). Also, it is well known that CD11b is expressed in both lymphoid and myeloid cells (Hanson \& GajlPeczalska, 1988). Though reactivity with these antibodies has been interpreted in the past as evidence of either lymphoid or myeloid lineage, recent studies suggest that one cannot assume absolute lineage-specificity for these antibodies. Therefore, the premise that the expression of $\mathrm{CD} 2$. CD19 and CD20 in AML or CD14 and CD11b in ALL represents evidence of biphenotypia may not be accurate. Only CD13 and $\mathrm{CD} 33$ have continued to be considered as myeloid-specific antigens. Further studies of normal marrows, fetal and adult, must be performed using multiparameter analysis to fully assess the normal immunologic sequence of haematopoietic development (Haynes et al, 1988; Uckun et al, 1989; LeBien et al, 1990; Grumayer et al, 1991). Only with this knowledge will we be able to truly assess biphenotypism.

Previous studies of biphenotypic leukaemia have suggested that these leukaemias may arise by malignant transformation of a pluripotent stem cell. The finding of a significantly greater percentage of biphenotypic cases with CD34 expression, compared to non-biphenotypic cases, supports the concept that this transformation process occurs at a step close to the haematopoietic progenitor cell. Similar conclusions have also been drawn from cytogenetic studies of acute leukaemia. Acute leukaemias that have either the t(9:22)(q34:q1 1) (Philadelphia chromsome) (Hirsch-Ginsberg et al, 1988; Hayashi et al, 1990b) or translocations involving 11q23 (Childs et al, 1988; Hayashi et al, 1990b) have been shown to have a high incidence of biphenotypic expression. These cytogenetic abnormalities are associated with both lymphoid and myeloid leukaemias suggesting that they reflect stem cell transformations. This 'dual' association may account for the 'promiscuous' lymphoid/myeloid immu- 
nophenotype frequently found in these leukaemias. Cytogenetic results from our study indeed did show that nine of the 31 clonal karyotypes contained the Philadelphia chromosome or an $11 \mathrm{q} 23$ translocation, confirming that these cytogenetic translocations are commonly found in biphenotypic leukaemia. Since both of these chromosomal abnormalities have clearly been shown to be independent, highrisk factors for acute leukaemia patients (Bloomfield et al, 1989), cytogenetic findings must also be considered when determining whether or not biphenotypic expression has any independent clinical significance in patients with acute leukaemia.

Other chromosomal aberrations identified in the biphenotypic leukaemias from our study also provoked some interesting speculation. Within the lymphoid antigen-positive AML group, classical myeloid-associated translocations, including $\mathrm{t}(8 ; 21), \mathrm{t}(15 ; 17)$, inv (16), trisomy $8,5 q-$, or $\mathrm{t}(9 ; 22)$, were identified in 13 of the 14 AML cases with cytogenetic abnormalities (Arthur et al, 1989; LeBeau, 1990). These translocations or deletions are typically associated with acute myelogenous leukaemias showing distinct morphologic and immunologic evidence of myeloid differentiation.

What then do the lymphoid-antigen-positive AML cases represent? The identification of these translocations so often associated with a myeloid phenotype, the morphologic evidence of differentiation (FAB-M2, M3, M4), and the probable lack of lineage-specificity of CD2, CD19 and CD20. raise the distinct possibility that these cases are simply cases of AML that are expressing uncommon 'lymphoid/myeloid'associated antigens. Ball et al (1991) also identified typical myeloid-associated translocations in lymphoid antigen-positive AML and showed that these leukaemias have a prognosis similar to or better than other AMLs. Thus, it may not be valid to look at this group of leukaemias as being truly biphenotypic. At the minimum, there is certainly the need to search for lymphoid-associated antigens on normal myeloid progenitor cells.

The cytogenetic findings in the myeloid antigen-positive ALL group, however, suggested a very different conclusion and raise some interesting diagnostic and biological questions. Some lymphoid-associated chromosomal abnormalities that can be seen in lymphoid leukaemias, such as hyperdiploidy, $6 \mathrm{q}-, \mathrm{t}(9 ; 22)$, and $11 \mathrm{q} 23$, were identified in 6/17 cases (Bloomfield et al, 1989). It was intriguing, however, to recognize several translocations within this myeloid antigen-positive ALL group that are more commonly associated with myeloid-type processes (Arthur et al, 1989; LeBeau, 1990). For example, trisomy 21 is a common finding in acute megakaryoblastic leukaemias (Johanson et al, 1990). Trisomy 8 is commonly found not only in acute myelogenous leukaemias, but also in myelodysplastic syndromes (LeBeau, 1990). Translocations involving 3q21 have been reported in myeloid leukaemias with abnormal megakaryopoiesis (Bernstein et al, 1982). The finding of these chromosomal abnormalities in cases with ALL morphology suggests that these leukaemias did indeed have dual-lineage heritage. As is summarized in a recent study (Drexler et al, 1991), adults with myeloid antigen-positive ALL may have a worse prognosis than those with conventional ALL. While biphenotypia is generally defined in immunologic terms, cytogenetic data may also be valuable when determining the cellular lineage of a leukaemic proliferation.

In summary, biphenotypic leukaemia continues to provoke more questions than answers. Some of these questions may be answered as more uniform laboratory diagnostic criteria are used and the spectrum of monoclonal antibody reactivity is more completely understood and standardized. Leukaemias with the Philadelphia chromosome or 11q23 translocations have clearly been associated with biphenotypic processes (Childs et al, 1988; Hirsch-Ginsberg et al, 1988; Hayashi et al, 1990b). Identification of other immunologic and cytogenetic subgroups will be essential in further characterizing and understanding these intriguing leukaemias and the cellular counterparts from which they arise.

\section{ACKNOWLEDGMENTS}

This study would not have been possible without the dedication and expertise of the Clinical Flow Cytometry Laboratory and Cytogenetic Laboratory. We also thank Shannon Fitzgerald for typing the manuscript.

\section{REFERENCES}

Akashi, K., Shibuya, T., Harada, M., Morioka, E., Oshima, K., Kimura, N., Takeshita, M., Kurokawa, M., Kikuchi, M. \& Niho, Y. (1990) Acute 'bilineal-biphenotypic' leukaemia. British Journal of Haematology, 74, 402-407.

Arthur, D.C., Berger, R., Golomb, H.M., Swansbury, G.J., Reeves, B.R., Alimena, G., Van Den Berghe, H., Bloomfield, C.D., de la Chapelle, A. \& Dewald, G.W. (1989) Sixth International Workshop on Chromosomes and Leukemia: The clinical significance of karyotypes in acute myelogenous leukemia. Cancer Genetics and Cytogenetics, 40, 203-216.

Bagwell, C.B. (1989) Clinical data analysis for flow cytometry. Flow Cytometry in Clinical Diagnosis (ed. by D. F. Keren), pp. 310-330. ASCP Press, Chicago.

Ball, E.D., Davis, R.B., Griffin, J.D., Mayer, R.J., Davey, F., Arthur, D.C., Wurster-Hill, D., Noll, W., El Ghetany, T., Allen, S.L., Rai, K., Lee, E.J., Schiffer, C.A. \& Bloomfield, C.D. (1991) Prognostic value of lymphocyte surface markers in acute myeloid leukemia. Blood, 10, 2242-2250.

Bernstein, R., Pinto, M. \& Mendelow, B. (1982) Chromosome 3 abnormalities in acute non-lymphocytic leukemia with abnormal thrombopoiesis. Blood, 60, 613-617.

Bloomfield, C.D., Secker-Walker, L.M., Goldman, A.I., Van Den Berghe, H., de la Chapelle, A., Ruutu, T., Alimena, G., Garson, O.M., Golomb, H.M. \& Rowley, J.D. (1989) Sixth International Workshop on Chromosomes and Leukemias: Six-year follow-up of the clinical significance of karyotype in acute lymphoblastic leukemia. Cancer Genetics and Cytogenetics, 40, 171-185.

Campos, L., Guyotat, D., Gentilhomme, O., Trielle, D., Fiere, D. \& Germain, D. (1987) Expression of a B-lymphoid differentiation antigen (CD19) on acute non-lymphoblastic leukaemia cells. European Journal of Haematology, 38, 220-224.

Childs, C.C., Hirsch-Ginsberg, C., Culbert, S.J., Ahearn, M., Reube, J., Trujillo, J.M., Cork, A., Walters, R.R., Freireich, E.J. \& Stass, S.A. (1988) Lineage heterogeneity in acute leukemias with the $t(4 ; 11)$ abnormality: implications for acute mixed lineage leukemia. Hematologic Pathology, 2, 145-157.

Civin, C.I., Banquerigo, M.L., Strauss, L.C. \& Loken, M.R. (1987) Antigenic analysis of hematopoiesis. VI. Flow cytometric charac- 
terization of $\mathrm{My}-10$ positive progenitor cells in normal human bone marrow. Experimental Hematology, 15, 10-17.

Cross, A.H., Goorha, R.M., Nuss, R., Behm, F.G., Murphy, S.B. Kalwinsky, D.K., Raimondi, S., Kitchingman, G.R. \& Mirro, J. (1988) Acute myeloid leukemia with T-lymphoid features: a distinct biologic and clinical entity. Blood, 72, 579-587.

Del Vecchio. L., Schiavone, E.M., Ferrara, F., Pace, E., Pardo, C.L., Pacetti, M.. Russo, M., Cirillo. D. \& Vacca, C. (1989) Immunodiagnosis of acute leukemia displaying ectopic antigens: proposal for a classification of promiscuous phenotypes. American Journal of Hematology. 31, 173-180.

Drexler, H.G., Menon, M., Sagawa, K., Tatsumi, E., Koshiba, H., Koishi, T., Minato, K., Sugimoto, T., Saito, M., Morita, M., Pauly, J.L.. Han, T.. Freeman, A.I., Messmore, H. \& Minowada, J. (1986) Phenotyping of malignant hematopoietic cells: analysis of 1200 cases of leukemia-lymphoma. Blut, 52, 99-109.

Drexler, H., Thiel, E. \& Ludwig, W.-D. (1991) Review of the incidence and clinical relevance of myeloid antigen-positive acute lymphoblastic leukemia. Leukemia, 5, 637-645.

Ferrara, F., De Rosa, C., Fasanaro, A., Mele, G., Finizio, O., Schiavone, E.M., Spada, O.A., Rametta, V. \& Del Vecchio, L. (1990) Myeloid antigen expression in adult acute lymphoblastic leukemia: clinicohematological correlations and prognostic relevance. Hematology and Pathology, 4, 93-98.

Gale, R.P. \& Ben-Bassat, I. (1987) Annotation: Hybrid acute leukaemia. British Journal of Haematology, 65, 261-264.

Gerhartz, H.H. \& Schmetzer, H. (1990) Detection of minimal residual disease in acute myeloid leukemia. Leukemia, 4, 508-516.

Greaves. M.F. \& Chan, L.C. (1986) Mixed lineage leukemia: the implications for hematopoietic differentiation. Blood, 68, 598603.

Greaves. M.F., Chan, L.C., Furley, A.J.W., Watt, S.M. \& Molgaard, H.V. (1986) Lineage promiscuity in hematopoietic differentiation and leukemia. Blood, 67, 1-11.

Grumayer, E.R., Griesinger, F., Hummell, D.S., Brunning, R.D. \& Kersey, J.H. (1991) Identification of novel B-lineage cells in human fetal bone marrow that coexpress CD7. Blood, 77, 64-68.

Hanson, C.A. \& Gajl-Peczalska, K.J. (1988) Monoclonal antibodies to lymphoreticular and myeloid antigens. Diagnostic Use of Monoclonal Antibodies in Clinical Immunohistochemistry (ed. by M. R. Wick and G. P. Siegal), pp. 147-226. Marcel Dekker, New York.

Harnden, D.G. \& Klinger, H.P. (eds) (1985) ISCN: An International System for Human Cytogenetic Nomenclature. Karger, Basel.

Hayashi, Y.. Pui, C.-H.. Behm, F.G., Fuchs, S.H., Raimondi, S.C.. Kitchingman. G.R., Mirro, J. \& Williams, D.L. (1990a) 14q32 translocations are associated with mixed-lineage expression in childhood acute leukemia. Blood, 76, 150-156.

Hayashi, Y., Sugita, K., Nakazawa, S., Abe, T., Kojima, S., Inaba, T. Hanada, R. \& Yamamoto, K. (1990b) Karyotypic patterns in acute mixed lineage leukemia. Leukemia, 4, 121-126.

Haynes, B.F., Martin, M.E., Kay, H.H. \& Kurtzberg, J. (1988) Early events in human T-cell ontogeny. Phenotypic characterization and immunohistologic localization of $\mathrm{T}$ cell precursors in early human fetal tissue. Journal of Experimental Medicine, 168, 10611080 .

Hirsch-Ginsberg, C., Childs, C., Chang, K.-S., Beran, M., Cork, A., Reuben, J., Freireich, E.J., Chang, L.C.M., Bollum, F.J., Trujillo, J. \& Stass, S.A. (1988) Phenotypic and molecular heterogeneity in Philadelphia chromosome-positive acute leukemia. Blood, 71 . $186-195$.

Johanson, B., Mertens. F., Heim, S., Kristoffersson, U., Mandahl, N., Nilsson, P.-G. \& Mitelman, F, (1990) Cytogenetic findings in acute megakaryoblastic leukemia. Cancer Genetics and Cytogenetics. 48. $119-123$.
Kaplan, S.S., Penchansky, L., Stolc, V., Contis, L. \& Krause, I.R. (1989) Immunophenotyping in the classification of acute leukemia in adults. Cancer, 63, 1520-1527.

Knapp, W. (ed.) (1989) Leukocyte Typing IV. Oxford University Press.

LeBeau, M.M. (1990) Chromosome abnormalities in hematologic malignant diseases. Mutation and the Environment, Part B (ed. by M. L. Mendelsohn and R. J. Albertine), p. 725. Wiley-Liss, New York.

LeBien, T.W.\& Villablanca. J.G. (1990) Ontogeny of normal human B-cell and T-cell precursors and its relation to leukemogenesis. Hematology/Oncology Clinics of North America, 4, 835-847.

LeBien, T.W., Wormann, B., Villablanca. J.G., Law, C., Steinberg. L.B., Shah, V.O. \& Loken, M.R. (1990) Multiparameter flow cytometric analysis of human fetal bone marrow B cells. Leukemia, $4,354-358$.

Maitreyan. V. \& Gale, R.P. (1989) What is hybrid acute leukemia? Leukemia Research, 13, 725-728.

Medeiros, C.J., Merrington, R.D., Gonzalaz, C.L., Jaffe, E.S. \& Cossman, I. (1991) My 4 antibody staining of non-Hodgkin's lymphomas. American Journal of Clinical Pathology, 95, 363-368.

McMichal, A.J. (ed.) (1987) Leukocyte Typing III. Oxford University Press.

Mirro, J., Kitchingman, G.R., Williams, D.L., Murphy, S.B., Zipf, T.F. \& Stass, S.A. (1986) Mixed lineage leukemia: the implications for hematopoietic differentiation. Blood, 68, 597-599.

Mirro. J., Zipf, T.F., Pui, C.-H., Kitchingman, G., Williams, D., Melvin. S. Murphy, S.B. \& Stass. S. (1985) Acute mixed lineage leukemia: clinicopathologic correlations and prognostic significance. Blood, 66, 1115-1123.

Perentesis, J., Ramsay, N.K.C., Brunning, R., Kersey, J.H. \& Filipovich. A.H. (1983) Biphenotypic leukemia: immunologic and morphologic evidence for a common lymphoid-myeloid progenitor in humans. Journal of Pediatrics, 102, 63-67.

Pui, C.-H., Behm, F.G., Singh, B., Rivera, G.K., Schell, M.J., Roberts, W.M., Crist, W.M. \& Mirro, J. (1990) Myeloid-associated antigen expression lacks prognostic value in childhood acute lymphoblastic leukemia treated with intensive multiagent chemotherapy. Blood. 75, 198-202.

Reinherz, E. (ed.) (1986) Leukocyte Typing III. Springer, New York. Ross, C.W., Stoolman, L.M., Schnitzer, B., Schlegelmilch, J. \& Hanson, C.A. (1990) Immunophenotypic aberrancy in adult acute lymphoblastic leukemia. American journal of Clinical Pathology, 94, 590-599.

Schmitt-Graff, A., Jurgens, H., Reifenhause, A., Schwamborn, D. \& Gobel, U. (1988) Childhood biphenotypic leukemia: detection of mixed lymphoid and myeloid populations in bone marrow specimens. Human Pathology, 19, 651-656.

Shapiro, H.M. (1988) Parameters and probes. Practical Flow Cytometry, 2nd edn (ed, by H. M. Shapiro), pp. 150-152. Alan R. Liss, New York.

Sheldon, S. \& Nichols, W.W. (1981) Comparison of the patterns of chromosomal late replication. Cytogenetics and Cell Genetics, 29. $40-50$.

Smith, L.J., Curtis, J.E., Messner, H.A., Senn, J.S., Furthmayr, H. \& McCulloch, E.A. (1983) Lineage infidelity in acute leukemia. Blood, 61, 1138-1145.

Sobol, R.E., Mick, R., Royston, I., Davey, F.R., Ellison, R.R., Newman, R., Cuttner, J., Griffin, J.D., Collins, H., Nelson, D.A. \& Bloomfield, C.D. (1987) Clinical importance of myeloid antigen expression in adult acute lymphoblastic leukemia. New England Journal of Medicine, 316, 1111-1117.

Stass, S. \& Mirro, J. (1985) Unexpected heterogeneity in acute leukemia: mixed lineages and lineage switch. Human Pathology, $16,864-866$.

Sulak. L.E., Clare, C.N., Morale, B.A., Hansen, K.L. \& Montiel, M.M. 
(1990) Biphenotypic acute leukemia in adults. American Journal of Clinical Pathology, 94, 54-58.

Terstappen, L.W. \& Loken. M.R. (1990) Myeloid cell differentiation in normal bone marrow and acute myeloid leukemia assessed by multi-dimensional flow cytometry. Annals of Cell Pathology, 2, 229-240.

Uckun, F.M., Muraguchi, A., Ledbetter, J.A., Kishimoto, T., O'Brien, R.T., Rolof, J.S., Gajl-Peczalska, K., Provisor, A. \& Koller, B. (1989)
Biphenotypic leukemic lymphocyte precursors in CD2 + CD19+ acute lymphoblastic leukemia and their putative normal counterparts in human fetal hematopoietic tissues. Blood, 73, 10001015.

Wiersma, S.R., Ortega, J., Sobel, E. \& Weinberg. K.I. (1991) Clinical importance of myeloid-antigen expression in acute lymphoblastic leukemia in childhood. New England Journal of Medicine. 324, 80()808 . 